\title{
Long-term outcomes of the 2-week schedule of hypofractionated radiotherapy for recurrent hepatocellular carcinoma
}

\author{
Jongmoo Park ${ }^{1,2}$, Jinhong Jung ${ }^{1}$, Daegeun Kim³ ${ }^{3}$ In-Hye Jung ${ }^{1}$, Jin-hong Park, Jong Hoon Kim¹,
} Sang-wook Lee ${ }^{1}$ and Sang Min Yoon ${ }^{1 *}$ (i)

\begin{abstract}
Background: The 2-week schedule of hypofractionated radiotherapy as a salvage treatment for hepatocellular carcinoma (HCC) has previously exhibited promising results; this study aimed to assess its long-term clinical outcomes in patients with recurrent HCC ineligible for curative treatments.

Methods: We retrospectively enrolled 77 patients (84 lesions) with HCC who were treated with hypofractionated radiotherapy between December 2008 and July 2013. Primary inclusion criteria were HCC unsuitable for curative treatments and HCC located within $2 \mathrm{~cm}$ of a critical normal organ. We administered 3.5-5 Gy/fraction for 2 weeks, resulting in a total dose of 35-50 Gy.

Results: The median follow-up period was 33.6 (range, 4.8-78.3) months. The 3- and 5-year overall survival rates were $52.3 \%$ and $40.9 \%$, respectively, and local control rates were $79.5 \%$ and $72.6 \%$ in all treated lesions, respectively. The 5 -year local control rate was better in the higher radiation dose group than in the lower radiation dose group (50 Gy: 79.7\% vs. $<50 \mathrm{~Gy}: 66.1 \%)$; however, the difference was not statistically significant $(P=0.493)$. We observed grade $\geq 3$ hepatic toxicity in $2(2.6 \%)$ patients and grade 3 gastrointestinal bleeding in 1 (1.3\%) patient. However, grade $\geq 4$ toxicity was not observed after hypofractionated radiotherapy.

Conclusions: The 2-week schedule of hypofractionated radiotherapy for recurrent HCC exhibited good local control and acceptable treatment-related toxicity during the long-term follow-up period. Thus, this fractionation schedule can be a potential salvage treatment option for recurrent HCC, particularly for tumors located close to a radiosensitive gastrointestinal organ.
\end{abstract}

Keywords: Hepatocellular carcinoma, Hypofractionated radiotherapy, Salvage therapy

\section{Background}

Liver cancer is the second leading cause of cancer-related death worldwide [1], and hepatocellular carcinoma (HCC) is the most common histological type of primary liver cancer [2]. Although surgical approaches and percutaneous ablation therapies are recommended as curative treatment options for $\mathrm{HCC}$, most patients with HCC are not suitable for these curative treatments. Typically, $<30 \%$ of patients undergo surgical treatment,

\footnotetext{
* Correspondence: drsmyoon@amc.seoul.kr

'Department of Radiation Oncology, Asan Medical Center, University of Ulsan College of Medicine, 88, Olympic-ro 43-gil, Songpa-gu, Seoul 05505, Republic of Korea

Full list of author information is available at the end of the article
}

owing to the extent of tumors, severity of underlying liver dysfunction, or limited resources. In addition, percutaneous ablation therapies are limited due to the location and/or size of HCC [3-6].

With remarkable recent technological advancements in radiotherapy, including four-dimensional (4D) computed tomography (CT), image-guided radiotherapy (IGRT), and respiratory-gated delivery, stereotactic body radiation therapy (SBRT) has emerged as an alternative treatment option for patients ineligible for the curative treatments [7-14]. A recent retrospective study demonstrated the potential role of SBRT for HCC by comparing the outcomes of radiofrequency ablation (RFA) and SBRT [15]. Despite the growing role of this high-dose

(c) The Author(s). 2018 Open Access This article is distributed under the terms of the Creative Commons Attribution 4.0 International License (http://creativecommons.org/licenses/by/4.0/), which permits unrestricted use, distribution, and reproduction in any medium, provided you give appropriate credit to the original author(s) and the source, provide a link to the Creative Commons license, and indicate if changes were made. The Creative Commons Public Domain Dedication waiver (http://creativecommons.org/publicdomain/zero/1.0/) applies to the data made available in this article, unless otherwise stated. 
radiotherapy, the use of SBRT for $\mathrm{HCC}$ located adjacent to radiosensitive gastrointestinal organs, such as the stomach, duodenum, esophagus, and/or large bowel, remains challenging because a high prescribed dose with a large fraction size can induce severe bleeding or perforation $[11-13,16]$. Furthermore, the majority of patients with HCC present with underlying liver cirrhosis, portal hypertension, and coagulopathy, all of which exacerbate the risk of gastrointestinal toxicity in comparison to patients without chronic liver diseases.

Thus, the fractionation scheme can be modified to reduce the probability of gastrointestinal toxicities. In our previous study, we adopted a 2-week schedule of hypofractionated radiotherapy to decrease the fraction size and reported the feasibility of this scheme for recurrent HCC located adjacent to critical organs [17]. The present study aimed to assess the long-term efficacy and safety of hypofractionated radiotherapy in patients with small recurrent HCC.

\section{Methods}

\section{Patient selection}

In this study, we retrospectively reviewed patients who underwent the 2-week schedule of hypofractionated radiotherapy for recurrent $\mathrm{HCC}$ at Asan Medical Center between December 2008 and July 2013. The detailed inclusion criteria for the use of hypofractionated radiotherapy has been described in our previous study [17]. The primary indication of this treatment scheme is the location of HCC within $2 \mathrm{~cm}$ of radiosensitive organs such as the stomach, duodenum, esophagus, or large bowel. HCC was diagnosed on the basis of histological confirmation and/or imaging criteria of the American Association for the Study of Liver Disease. This retrospective study was approved by the Institutional Review Board of the Asan Medical Center and the requirement for informed consent was waived because of the retrospective nature of this study.

\section{Radiotherapy}

All patients were immobilized in the supine position using a vacuum cushion. Then, each patient underwent 4D CT simulation with free breathing using a 16-slice CT (GE LightSpeed RT 16; GE Healthcare, Waukesha, WI, USA). The breathing pattern was assessed using the Real-time Position Management System (Varian Medical System, Palo Alto, CA, USA). The CT slice thickness was set to $2.5 \mathrm{~mm}$. An intravenous contrast agent was used to improve the accuracy of target volume delineation. 4D imaging software (Advantage 4D; GE Healthcare) was used to sort 4D-CT datasets from 10-phase bins corresponding to the respiratory phase. One week before the CT simulation, three gold fiducial markers (CIVICO Medical Solution, Kalona, IA,
USA) were implanted into the liver parenchyma under ultrasound guidance, except for those with surgical clips or compact iodized oil that could be used as a fiducial marker on cone-beam $\mathrm{CT}$ and fluoroscopy [18].

The gross tumor volume (GTV) was defined as an arterial enhancing lesion with washout on diagnostic dynamic enhanced CT and/or magnetic resonance imaging (MRI) at the end-expiratory phase of simulation CT imaging. The internal target volume (ITV) was defined as the summation of individual GTVs in the gated respiratory interval (mostly 30-70\%) or in an entire respiratory cycle. The planning target volume (PTV) was obtained by expanding $0.5 \mathrm{~cm}$ from the ITV in all directions. The normal liver, esophagus, stomach duodenum, colon, kidneys, and spinal cord were contoured for dose constraint for organs at risk.

Three-dimensional conformal radiotherapy technique was used to determine radiation ports using a planning system (Eclipse; Varian Medical Systems) and the most actual beam delivery was performed with a respiratorygated delivery technique. Of note, the total dose to the PTV was 35-50 Gy, administered in 10 fractions (5 fractions/week) and the fraction size was determined according to the maximum dose in adjacent radiosensitive organs. Over $95 \%$ of the PTV received $100 \%$ of the prescription dose and the chosen isodose covering PTV was between 85 and 90\%, which was normalized to the center of the PTV. In this study, the dose constraints for normal organs were as follows: no more than $25 \%$ of the normal liver volume would receive over $50 \%$ of the prescribed dose; maximum dose to the colon and esophagus should not exceed $3.5 \mathrm{~Gy} /$ fraction; and the dose to the stomach, duodenum, and spinal cord should not exceed $2.5 \mathrm{~Gy} /$ fraction.

\section{Evaluation and statistical analysis}

During hypofractionated radiotherapy, all patients were examined to assess the acute toxicity. After the completion of treatment, the medical history was obtained and physical examinations, complete blood counts, biochemical profiles, coagulation tests, tumor markers, and CT or MRI were performed at 2- or 3-month intervals. Notably, the toxicity, related to constitutional symptoms, gastrointestinal effects, and laboratory findings during and after the treatment was assessed according to the Common Terminology Criteria for Adverse Events (version 4.03). In addition, the deterioration of the Child-Pugh score (increased by $\geq 2$ ) in the absence of tumor progression within 3 months after radiotherapy was evaluated [19].

The tumor response after radiotherapy was evaluated according to the Response Evaluation Criteria in Solid Tumor (RECIST, version 1.1) at 3 months after the 
completion of radiotherapy. Local failure was defined as the recurrence of a treated lesion, and progression was defined as any type of recurrence, including intrahepatic recurrence or extrahepatic metastasis for the progression-free survival (PFS). The overall survival (OS) and PFS were estimated from the date of the start of radiotherapy to the date of death or the last follow-up and to the date of death, tumor recurrence, or last follow-up, respectively, using the Kaplan-Meier method. Both univariate and multivariate analyses were performed using the Cox proportional hazards model to illustrate the association of variables with survival outcomes. The backward elimination Cox's regression was used to select the principal risk factors in the multivariate model. All tests were two-sided, and $P<0.05$ was considered statistically significant. All statistical analyses were performed using the SPSS software (version 21; IBM SPSS Statistics, Armonk, NY, USA).

Table 1 Patient characteristics

\begin{tabular}{|c|c|}
\hline Variables & Number of patients (\%) \\
\hline \multicolumn{2}{|l|}{ Sex } \\
\hline Male & $45(58.4)$ \\
\hline Female & $32(41.6)$ \\
\hline \multicolumn{2}{|l|}{ Age (years) } \\
\hline Median & 62 \\
\hline Range & $42-88$ \\
\hline \multicolumn{2}{|c|}{ ECOG performance status } \\
\hline 0 & $60(77.9)$ \\
\hline 1 & $11(14.3)$ \\
\hline $2-3$ & $6(7.8)$ \\
\hline \multicolumn{2}{|l|}{ Child-Pugh class } \\
\hline A & $56(72.7)$ \\
\hline B & $21(27.3)$ \\
\hline \multicolumn{2}{|l|}{ Viral etiology } \\
\hline Hepatitis B virus & $51(66.2)$ \\
\hline Hepatitis C virus & $14(18.2)$ \\
\hline Others & $12(15.6)$ \\
\hline \multicolumn{2}{|c|}{ Alpha-fetoprotein (ng/mL) } \\
\hline$<200$ & $58(75.3)$ \\
\hline$\geq 200$ & $19(24.7)$ \\
\hline \multicolumn{2}{|c|}{ Tumor size $(\mathrm{cm})(n=84)$} \\
\hline Median & 2.4 \\
\hline Range & $0.8-5.6$ \\
\hline$\leq 3 \mathrm{~cm}$ & $59(70.2)$ \\
\hline$>3 \mathrm{~cm}$ & $25(29.8)$ \\
\hline
\end{tabular}

ECOG Eastern Cooperative Oncology Group

\section{Results}

\section{Patients and treatment}

During the study period, 120 patients with recurrent HCC were treated with the hypofractionated radiotherapy. Of these, 43 patients were excluded from the analysis because of the following reasons: a history of previous radiotherapy to the liver $(n=22)$, presence of vascular invasion $(n=10)$, multiple viable HCCs outside the radiation field $(n=5)$, presence of extrahepatic metastasis $(n=3)$, and lost to follow-up just after the completion of radiotherapy $(n=3)$. The remaining 77 patients (84 lesions) fulfilled the inclusion criteria and were enrolled in this study (Table 1, Additional files 1 and 2). The median tumor size was $2.4 \mathrm{~cm}$ and approximately $30 \%$ of lesions were $>3 \mathrm{~cm}$ in size.

Before receiving hypofractionated radiotherapy, all patients had received various previous therapies, including hepatic resection, RFA, percutaneous ethanol injection, transarterial chemoembolization (TACE), or a combination of these treatments (Table 2). The median number of previous treatment sessions was 4 (range, 1-19). In addition, 50 (59.5\%) lesions received a total dose of $50 \mathrm{~Gy}$, and the remaining lesions were administered 40 or 35 Gy according to the maximum dose in adjacent normal organs as mentioned earlier. Four patients received radiotherapy using a volumetric-modulated arc

Table 2 Treatment characteristics of 84 lesions

\begin{tabular}{ll}
\hline Variables & Number of patients (\%) \\
\hline Previous treatment $(n=77)$ & $41(53.2)$ \\
TACE & $17(22.1)$ \\
TACE + RFA & $3(3.9)$ \\
TACE + PEI & $1(1.3)$ \\
TACE + RFA + PEI & $6(7.8)$ \\
TACE + Resection & $6(7.8)$ \\
TACE + Resection + RFA & $1(1.3)$ \\
TACE + Resection + PEl & $1(1.3)$ \\
TACE + Resection + RFA + PEI & $1(1.3)$ \\
RFA & \\
Radiation dose & $7(8.3)$ \\
35 Gy/10 fractions & $27(32.2)$ \\
40 Gy/10 fractions & $50(59.5)$ \\
50 Gy/10 fractions & \\
Radiotherapy plan & $80(95.2)$ \\
Three-dimensional conformal radiotherapy & $4(4.8)$ \\
Volumetric-modulated arc therapy & \\
Respiratory-gated beam delivery & $79(94.0)$ \\
Yes & $5(6.0)$ \\
No &
\end{tabular}

TACE transarterial chemoembolization, RFA radiofrequency ablation, $P E I$ percutaneous ethanol injection 


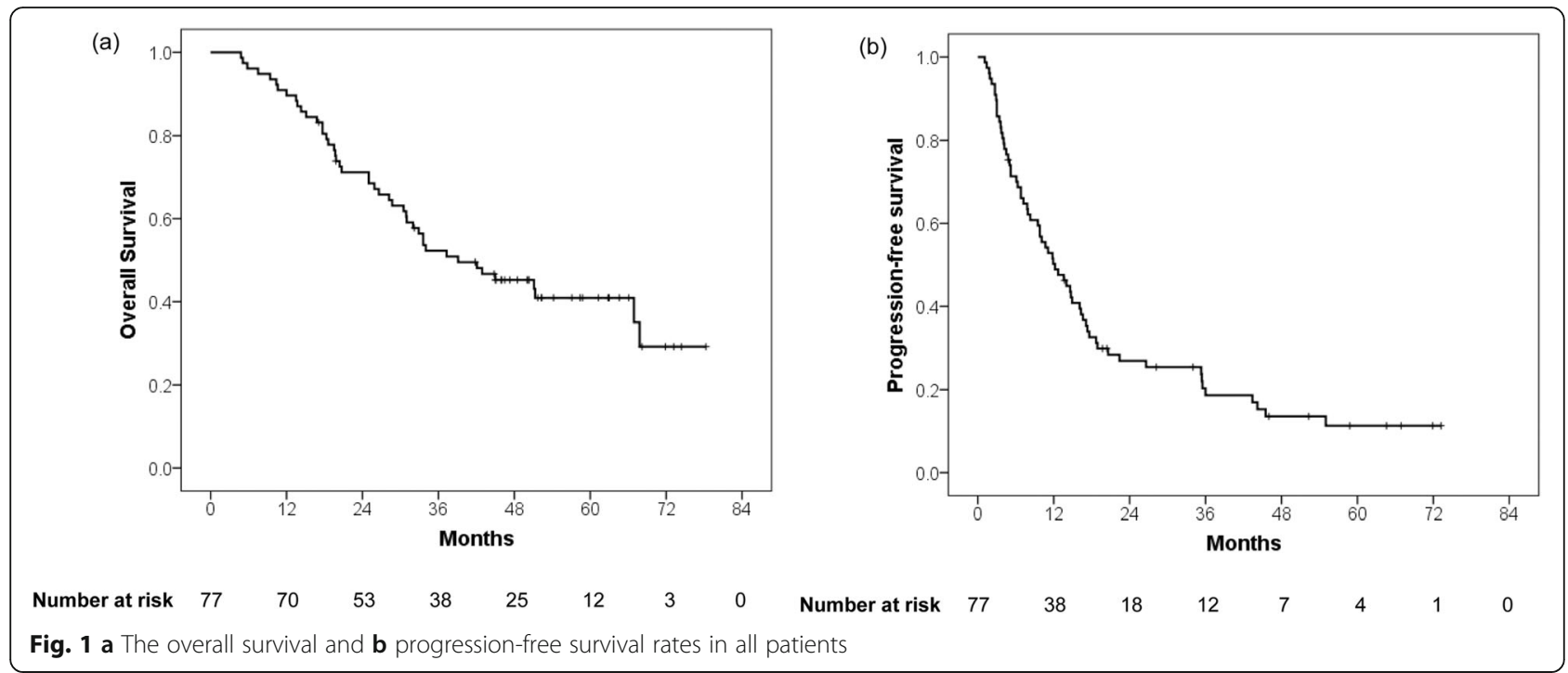

therapy technique to address stomach and duodenal constraints. Most lesions (94\%) were treated using the respiratory-gated technique during radiotherapy (Table 2).

\section{Radiotherapy response and survival outcomes}

Three months after the completion of radiotherapy, complete response, partial response, stable disease, and progressive disease were attained in 33 (39.3\%), 27 (32.1\%), 23 (27.4\%), and $1(1.2 \%)$ lesions, respectively. The median follow-up period for all patients and surviving patients was 33.6 (range, 4.8-78.3) and 52.0 (range, 17.1-78.3) months, respectively. At the time of analysis, 45 patients died, and 32 patients remained alive. The 3- and 5-year OS rates were $52.3 \%$ and $40.9 \%$, respectively (Fig. 1a). In the multivariate analysis, the number of previous treatment was a significant prognostic factor for the OS (hazard ratio $[\mathrm{HR}]=1.08$; 95\% confidence interval $[\mathrm{CI}], 1.01-1.16 ; P=0.029$; Table 3). Sixty-four $(83.1 \%)$ patients experienced tumor recurrences after radiotherapy. The most common failure pattern was the development of a new HCC in the liver (89.1\%).
The 3- and 5-year PFS rates were $18.7 \%$ and $11.3 \%$, respectively, in all patients (Fig. 1b).

\section{Local control rates}

The 3- and 5-year local control rates in all lesions were $79.5 \%$ and $72.6 \%$, respectively (Fig. 2a). Based on the prescribed dose, the 5-year local control rate was better in the higher radiation dose group than the lower radiation dose group (50 Gy: 79.7\% vs. < 50 Gy: 66.1\%); however, the difference was not statistically significant $(\mathrm{HR}=0.70$; 95\% CI, 0.25-1.94; $P=0.493$; Fig. 2b). There was no other clinical parameter that was associated with local tumor control after hypofractionated radiotherapy (Additional file 3: Table S1).

\section{Treatment-related toxicity}

All patients received planned radiotherapy without any interruptions. Table 4 summarizes treatment-related toxicities in this study. Although the worsening of the biochemical blood tests, such as transaminase, bilirubin, or

Table 3 Factors affecting the overall survival after hypofractionated radiotherapy

\begin{tabular}{|c|c|c|c|c|c|c|}
\hline \multirow[t]{2}{*}{ Variables } & \multicolumn{3}{|c|}{ Univariate analysis } & \multicolumn{3}{|c|}{ Multivariate analysis } \\
\hline & $\mathrm{HR}$ & $95 \% \mathrm{Cl}$ & $P$ value & $\mathrm{HR}$ & $95 \% \mathrm{Cl}$ & $P$ value \\
\hline Sex & 1.05 & $0.60-1.83$ & 0.876 & & & \\
\hline Age & 1.02 & $0.99-1.05$ & 0.144 & 1.03 & $0.99-1.06$ & 0.080 \\
\hline ECOG PS (0-1 vs. 2-3) & 1.30 & $0.51-3.28$ & 0.581 & & & \\
\hline Tumor size & 1.23 & $0.96-1.58$ & 0.101 & 1.24 & $0.95-1.61$ & 0.119 \\
\hline Alpha-fetoprotein $\left(\log _{10}\right)$ & 1.24 & $0.92-1.67$ & 0.154 & 1.17 & $0.86-1.59$ & 0.323 \\
\hline Child-Pugh class & 1.20 & $0.65-2.21$ & 0.570 & & & \\
\hline Number of previous treatment sessions & 1.10 & $1.03-1.17$ & 0.007 & 1.08 & $1.01-1.16$ & 0.029 \\
\hline Dose (50 Gy vs. < 50 Gy) & 1.03 & $0.98-1.08$ & 0.310 & & & \\
\hline
\end{tabular}



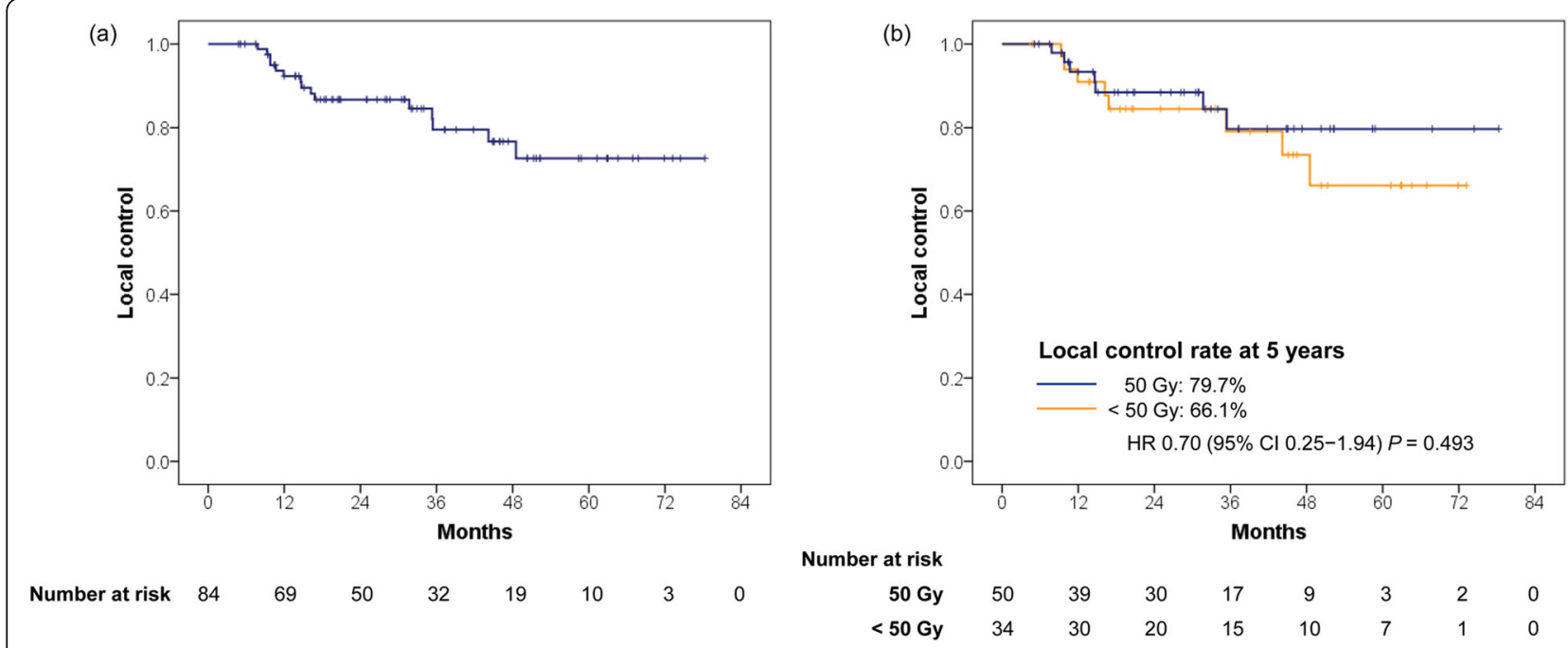

Fig. 2 a The local control rate and $\mathbf{b}$ local control rates according to the prescribed dose in all lesions

alkaline phosphatase, was the most common toxicity, however, the incidence of grade $\geq 3$ toxicity was only $2.6 \%$ ( 2 patients); these elevated transaminases spontaneously normalized within 3 months with supportive care only. An elevation of the Child-Pugh score $\geq 2$ without tumor progression was observed in 4 (5.2\%) patients. The most common acute constitutional symptoms other than hepatic toxicities were fatigue, anorexia, and nausea; however, these toxicities were mostly grade 1 and resolved immediately after radiotherapy.

During the follow-up period, one patient who was administered 50 Gy experienced grade 3 gastrointestinal bleeding after 5 months of radiotherapy. The tumor was located in segment 2, and the distance between the

Table 4 Treatment-related toxicity

\begin{tabular}{llllll}
\hline Adverse events & \multicolumn{5}{l}{ Number of patients } \\
\cline { 2 - 6 } & Grade 1 & Grade 2 & Grade 3 & Grade 4 & Grade 5 \\
\hline Acute & 15 & 2 & 0 & 0 & 0 \\
Fatigue & 8 & 2 & 0 & 0 & 0 \\
Anorexia & 5 & 3 & 0 & 0 & 0 \\
Abdominal Pain & 5 & 0 & 0 & 0 & 0 \\
Nausea & 6 & & & & \\
Biochemical & & 3 & 2 & 0 & 0 \\
$\quad$ Transaminase & 30 & 3 & 0 & 0 & 0 \\
$\quad$ Bilirubin & 19 & 13 & 0 & 0 & 0 \\
$\quad$ Alkaline & 16 & 0 & & & \\
phosphatase & & & 0 & 0 & 0 \\
Late & 1 & 0 & 0 & 0 & 0 \\
Rib fracture & 1 & 0 & 0 & 0 & 0 \\
Biliary stricture & 1 & 0 & 1 & & \\
Gastrointestinal & 0 & & & &
\end{tabular}

stomach antrum and $\mathrm{HCC}$ was $1.5 \mathrm{~cm}$ on simulation CT. Despite the maximum dose to the stomach being 30 Gy in the treatment planning, endoscopy revealed active bleeding foci in the stomach antrum. The patient received supportive care, including blood transfusion and endoscopic hemostasis, and subsequently recovered well. Regarding other late toxicities, a rib fracture (grade 1, 15 months after radiotherapy) and a biliary stricture (grade 1, 11 months after radiotherapy) developed; however, these toxicities did not require specific treatment. Of note, no patients experienced grade 4 or 5 toxicity after hypofractionated radiotherapy.

\section{Discussion}

With the development of radiotherapy technology, SBRT (usually $\leq 5-6$ fractions) has found utility in the management of small HCC when curative treatment modalities cannot be applied [7, 8, 10-13]. Although high-dose radiation offers the biological advantage of contributing to the local tumor control, radiation-induced toxicity is a concern in cases of HCC adjacent to radiosensitive gastrointestinal organs. Besides, the majority of patients with HCC present with an underlying chronic liver disease, which could exacerbate the risk of gastrointestinal toxicities, owing to the hypothesis of the impairment of the mucosal defense mechanism [20]. Several studies investigating SBRT using total doses of $25-60$ Gy in 3-5 fractions reported grade $\geq 3$ gastrointestinal toxicities, such as bleeding or perforation [11-13, 16, 21, 22]. Jang et al. reported that $5(6 \%)$ patients whose tumors were located in the vicinity of the gastrointestinal tract experienced grade 3 or 4 gastrointestinal ulcers or perforations after 3-fraction SBRT for HCC [13]. In addition, Huertas et al. reported three (3.9\%) gastric or colonic ulcers, although they used alternative SBRT fractionation 


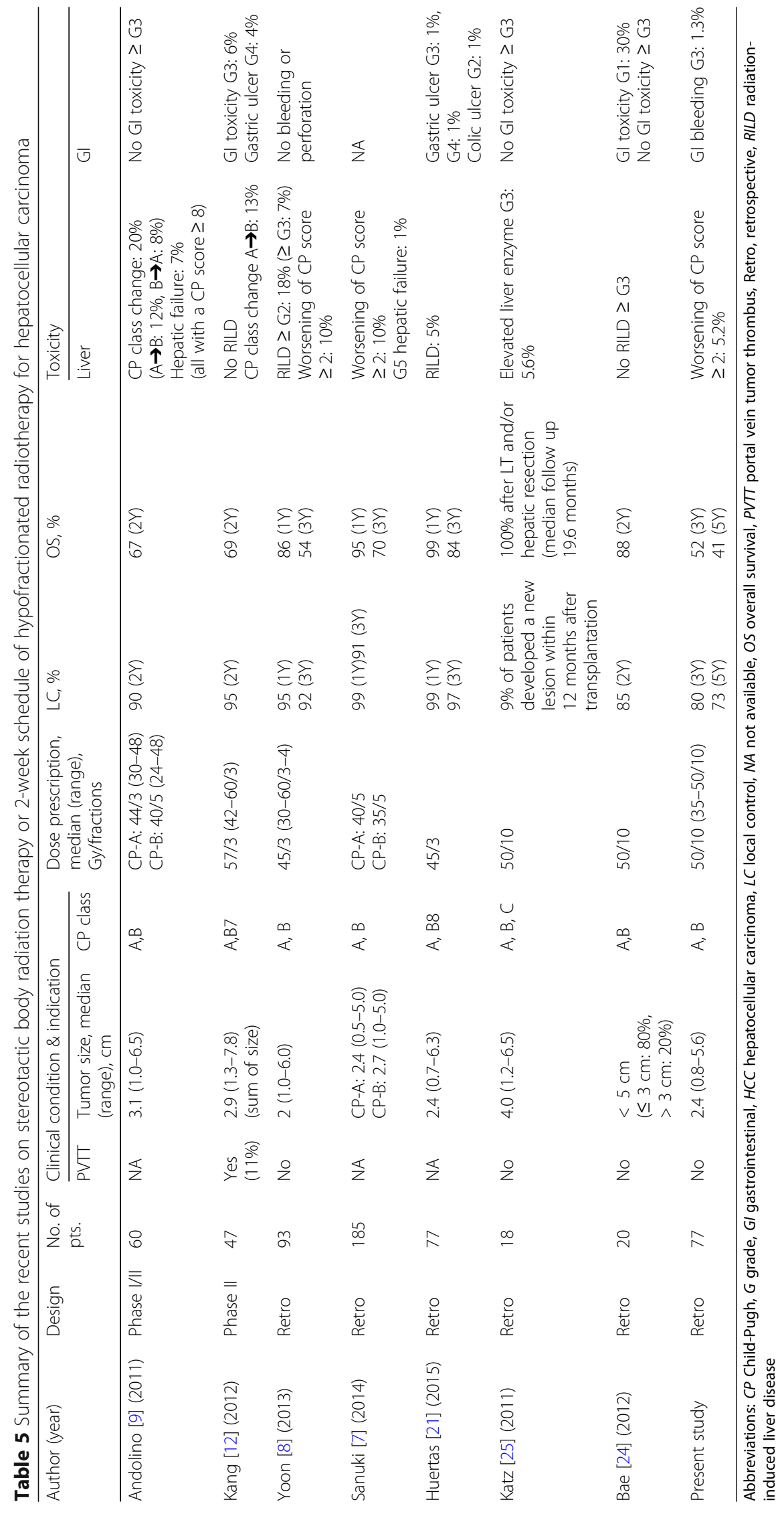


protocols of 50 Gy in 4 or 5 fractions, rather than 45 Gy in 3 fractions, in case of tumors located adjacent to the gastrointestinal tract [21]. Although SBRT exhibits a promising local control in patients with HCC, a severe complication should be considered in patients with $\mathrm{HCC}$ adjacent to gastrointestinal organs even if the overall incidence is relatively low.

In the present study, the 2-week schedule of hypofractionated radiotherapy for recurrent HCC located adjacent to radiosensitive gastrointestinal organs yielded an encouraging long-term local control rate $(72.6 \%$ at 5 years) with acceptable toxicity. Regarding the local tumor control, this fractionation scheme exhibited a marginally lower local control rate compared with that of SBRT delivering higher doses with 3-5 fractions $[7,8,11,12,21]$. However, the patients in our study had no effective local treatment options, such as resection, transplantation, RFA, or TACE, owing to the complicated clinical situations. Moreover, their tumors were located adjacent to the stomach, duodenum, esophagus, or colon; this could be another hindrance in deciding the optimal treatment. When SBRT using 3-5 fractions cannot be performed due to the imminent risk of gastrointestinal toxicity, this 2-week schedule radiotherapy could be safely implemented. In this study, all lesions located within $2 \mathrm{~cm}$ from gastrointestinal organs were treated with a smaller fractional dose (3.5-5 Gy), and only 1 (1.3\%) patient experienced grade 3 gastrointestinal bleeding, which, however, recovered with conservative management. Table 5 summarizes the clinical outcomes of the recent studies of SBRT or 2-week schedule of hypofractionated radiotherapy for HCC.

Iwata et al. assessed the outcomes of hypofractionated radiotherapy (50 or 55 Gy in 10 fractions) for $\mathrm{HCC}(n=$ 6) and liver metastasis $(n=12)$ and reported the 1-year local control rate for HCC as $100 \%$ without any gastrointestinal toxicity based on the dose constraints for the stomach, duodenum, and colon as 40 Gy in 10 fractions for $\leq 10$ cc [23]. Likewise, Bae et al. reported the results of 50 Gy radiotherapy in 10 fractions as a salvage treatment for recurrent small HCC [24], although this study had limited experience due to enrollment of only patients with HCC not adjacent to the stomach or duodenum, no patient developed grade $\geq 3$ toxicity with the 2 -year local control rate of $85 \%$. In addition, Katz et al. used a median total dose of 50 Gy in 10 fractions of radiotherapy and reported an excellent in-field control with minimal side effects as a bridge to transplantation for HCC [25]. Compared with all other previous studies mentioned earlier, our study discusses two issues. First, all the previous studies enrolled small study cohorts and did not present the long-term local control rate or treatment-related toxicity because the follow-up time after radiotherapy was relatively short. However, our study provided the long-term local control rate $(72.6 \%$ at 5 years) with a low incidence (1.3\%) of gastrointestinal complication as mentioned earlier. Second, in this study, the primary indication of this fractionation scheme is the $\mathrm{HCC}$ located $<2 \mathrm{~cm}$ of the gastrointestinal organs; the prescription dose ranged 35-50 Gy in 10 fractions depending on the dose constraints for normal organs in comparison to previous studies mentioned earlier.

As hypofractionated radiotherapy was performed for patients with recurrent HCCs after various sessions of previous treatments in this study, the 5-year OS rate was relatively low $(40.9 \%)$ compared with the outcomes of surgical resection or RFA in patients with early-stage HCC. Multivariate analysis revealed that the number of previous treatment sessions was a significant prognosticator for the OS $(\mathrm{HR}=1.08 ; 95 \% \mathrm{CI}, 1.01-1.16 ; P=$ 0.029). Bae et al. reported that patients receiving fewer than two courses of local treatment exhibited a trend toward superior intrahepatic control compared with patients who had received more than two courses of local treatments [24]. Although they did not compare the OS based on the number of previous treatments, frequent intrahepatic recurrences may affect the survival outcomes in patients with recurrent HCC.

This study has some limitations. First, being a retrospective study, it might encompass a few potential risks, including selection bias and missing data. Second, in our patient cohort, there was heterogeneity in the treatment before and after radiotherapy, owing to the frequent incidence of recurrence, which could be associated with the overall patient's outcomes. Nevertheless, this study enrolled a relatively large number of patients with HCC located adjacent to gastrointestinal tracts and also presented the long-term follow-up clinical outcomes.

\section{Conclusions}

The 2-week schedule of hypofractionated radiotherapy is safe and effective, although the local control rate was not as high as that reported in recent studies on SBRT using 3-5 fractions. Overall, this radiotherapy scheme can be a potential alternative treatment option in patients with $\mathrm{HCC}$ located in the vicinity of the radiosensitive gastrointestinal tract when shortcourse SBRT is not feasible.

\section{Additional files}

Additional file 1: Raw data of the patients. Patient characteristics and follow-up data exists. (XLSX $16 \mathrm{~kb}$ )

Additional file 2: Raw data of the treated lesions. Lesion characteristics and follow-up data exists. (XLSX $15 \mathrm{~kb}$ )

Additional file 3: Table S1. Factors associated with the local tumor control after hypofractionated radiotherapy. (DOCX $19 \mathrm{~kb}$ ) 


\section{Abbreviations}

4D: 4-dimensional; Cl: Confidence interval; CT: Computed tomography; GTV: Gross tumor volume; HCC: Hepatocellular carcinoma; HR: Hazard ratio; IGRT: Image-guided radiation therapy; ITV: Internal target volume; MRI: Magnetic resonance image; OS: Overall survival; PFS: Progression-free survival; PTV: Planning target volume; RECIST: Response Evaluation Criteria in Solid Tumors; RFA: Radiofrequency ablation; SBRT: Stereotactic body radiation therapy; TACE: Transarterial chemoembolization

\section{Acknowledgements}

Not applicable.

\section{Funding}

None.

\section{Availability of data and materials}

All data analyzed during this study are included in the additional files.

\section{Authors' contributions}

All authors have full access to all data and take responsibility for the accuracy of the data analysis. All authors approved the final version for submission. Conceived and designed the experiments: JP SMY JJ IHJ. Performed the experiments: JP SMY JHK JHP JJ IHJ SWL. Analyzed the data: JP SMY DK JJ. Contributed reagents/materials/analysis tools: JP SMY JHK JHP JJ DK IHJ SWL. Wrote the paper: JP SMY

\section{Ethics approval and consent to participate}

This study was approved by the Institutional Review Board of Asan Medical Center, and informed consent was waived because of the retrospective nature of the study. The datasets used and/or analyzed during the current study are available from the corresponding author on reasonable request.

\section{Consent for publication}

Not applicable.

\section{Competing interests}

The authors declare that they have no competing interests.

\section{Publisher's Note}

Springer Nature remains neutral with regard to jurisdictional claims in published maps and institutional affiliations.

\section{Author details}

'Department of Radiation Oncology, Asan Medical Center, University of Ulsan College of Medicine, 88, Olympic-ro 43-gil, Songpa-gu, Seoul 05505, Republic of Korea. ${ }^{2}$ Department of Radiation Oncology, Chungbuk National University Hospital, Cheongju 28644, Republic of Korea. ${ }^{3}$ University of Ulsan College of Medicine, 88, Olympic-ro 43-gil, Songpa-gu, Seoul 05505, Republic of Korea.

\section{Received: 2 May 2018 Accepted: 15 October 2018}

\section{Published online: 26 October 2018}

\section{References}

1. Ferlay J, Soerjomataram I, Dikshit R, Eser S, Mathers C, Rebelo M, et al. Cancer incidence and mortality worldwide: sources, methods and major patterns in GLOBOCAN 2012. Int J Cancer. 2015;136:E359-86.

2. El-Serag HB. Epidemiology of viral hepatitis and hepatocellular carcinoma. Gastroenterology. 2012;142:1264-73 e1.

3. Bruix J, Sherman M. American Association for the Study of Liver Diseases. Management of hepatocellular carcinoma: an update. Hepatology. 2011;53: 1020-2.

4. Dhir M, Melin AA, Douaiher J, Lin C, Zhen WK, Hussain SM, et al. A review and update of treatment options and controversies in the management of hepatocellular carcinoma. Ann Surg. 2016;263:1112-25.

5. Bruix J, Sherman M, Llovet JM, Beaugrand M, Lencioni R, Burroughs AK, et al. Clinical management of hepatocellular carcinoma. Conclusions of the Barcelona-2000 EASL conference. European Association for the Study of the Liver J Hepatol. 2001:35:421-30.

6. Yu SJ. A concise review of updated guidelines regarding the management of hepatocellular carcinoma around the world: 2010-2016. Clin Mol Hepatol. 2016:22:7-17.
7. Sanuki N, Takeda A, Oku Y, Mizuno T, Aoki Y, Eriguchi T, et al. Stereotactic body radiotherapy for small hepatocellular carcinoma: a retrospective outcome analysis in 185 patients. Acta Oncol. 2014;53:399-404.

8. Yoon SM, Lim YS, Park MJ, Kim SY, Cho B, Shim JH, et al. Stereotactic body radiation therapy as an alternative treatment for small hepatocellular carcinoma. PLoS One. 2013;8:e79854.

9. Andolino DL, Johnson CS, Maluccio M, Kwo P, Tector AJ, Zook J, et al. Stereotactic body radiotherapy for primary hepatocellular carcinoma. Int J Radiat Oncol Biol Phys. 2011;81:e447-53.

10. Bujold A, Massey CA, Kim JJ, Brierley J, Cho C, Wong RK, et al. Sequential phase I and II trials of stereotactic body radiotherapy for locally advanced hepatocellular carcinoma. J Clin Oncol. 2013;31:1631-9.

11. Huang WY, Jen YM, Lee MS, Chang LP, Chen CM, Ko KH, et al. Stereotactic body radiation therapy in recurrent hepatocellular carcinoma. Int J Radiat Oncol Biol Phys. 2012;84:355-61.

12. Kang JK, Kim MS, Cho CK, Yang KM, Yoo HJ, Kim JH, et al. Stereotactic body radiation therapy for inoperable hepatocellular carcinoma as a local salvage treatment after incomplete transarterial chemoembolization. Cancer. 2012; 118:5424-31.

13. Jang WI, Kim MS, Bae SH, Cho CK, Yoo HJ, Seo YS, et al. High-dose stereotactic body radiotherapy correlates increased local control and overall survival in patients with inoperable hepatocellular carcinoma. Radiat Oncol. 2013;8:250.

14. Rim $\mathrm{CH}$, Seong J. Application of radiotherapy for hepatocellular carcinoma in current clinical practice guidelines. Radiat Oncol J. 2016;34:160-7.

15. Wahl DR, Stenmark MH, Tao Y, Pollom EL, Caoili EM, Lawrence TS, et al. Outcomes after stereotactic body radiotherapy or radiofrequency ablation for hepatocellular carcinoma. J Clin Oncol. 2016;34:452-9.

16. Louis C, Dewas S, Mirabel X, Lacornerie T, Adenis A, Bonodeau F, et al. Stereotactic radiotherapy of hepatocellular carcinoma: preliminary results. Technol Cancer Res Treat. 2010;9:479-87.

17. Park JH, Yoon SM, Lim YS, Kim SY, Shim JH, Kim KM, et al. Two-week schedule of hypofractionated radiotherapy as a local salvage treatment for small hepatocellular carcinoma. J Gastroenterol Hepatol. 2013;28:1638-42.

18. Park SH, Won HJ, Kim SY, Shin YM, Kim PN, Yoon SM, et al. Efficacy and safety of ultrasound-guided implantation of fiducial markers in the liver for stereotactic body radiation therapy. PLoS One. 2017:12:e0179676.

19. Pan CC, Kavanagh BD, Dawson LA, Li XA, Das SK, Miften M, et al. Radiationassociated liver injury. Int J Radiat Oncol Biol Phys. 2010;76:S94-100.

20. Kitano S, Dolgor B. Does portal hypertension contribute to the pathogenesis of gastric ulcer associated with liver cirrhosis? J Gastroenterol. 2000:35:79-86.

21. Huertas A, Baumann AS, Saunier-Kubs F, Salleron J, Oldrini G, Croise-Laurent V, et al. Stereotactic body radiation therapy as an ablative treatment for inoperable hepatocellular carcinoma. Radiother Oncol. 2015;115:211-6.

22. Bae SH, Kim MS, Cho CK, Kim KB, Lee DH, Han CJ, et al. Feasibility and efficacy of stereotactic ablative radiotherapy for Barcelona clinic liver Cancer-C stage hepatocellular carcinoma. J Korean Med Sci. 2013;28:213-9.

23. Iwata H, Shibamoto Y, Hashizume C, Mori Y, Kobayashi T, Hayashi N, et al. Hypofractionated stereotactic body radiotherapy for primary and metastatic liver tumors using the novalis image-guided system: preliminary results regarding efficacy and toxicity. Technol Cancer Res Treat. 2010;9:619-27.

24. Bae SH, Park HC, Lim DH, Lee JA, Gwak GY, Choi MS, et al. Salvage treatment with hypofractionated radiotherapy in patients with recurrent small hepatocellular carcinoma. Int J Radiat Oncol Biol Phys. 2012;82:e603-7.

25. Katz AW, Chawla S, Qu Z, Kashyap R, Milano MT, Hezel AF. Stereotactic hypofractionated radiation therapy as a bridge to transplantation for hepatocellular carcinoma: clinical outcome and pathologic correlation. Int J Radiat Oncol Biol Phys. 2012;83:895-900. 